\title{
Cross-dimensional perceptual selectivity
}

\author{
JAN THEEUWES \\ TNO Institute for Perception, Soesterberg, The Netherlands
}

\begin{abstract}
Three visual search experiments tested whether top-down selectivity toward particular stimulus dimensions is possible during preattentive parallel search. Subjects viewed multielement displays in which two salient items, each unique in a different dimension-that is, color and intensity (Experiment 1) or color and form (Experiments 2 and 3)-were simultaneously present. One of the dimensions defined the target; the other dimension served as distractor. The results indicate that when search is performed in parallel, top-down selectivity is not possible. These findings suggest that preattentive parallel search is strongly automatic, because it satisfies both the load-insensitivity and the unintentionality criteria of automaticity.
\end{abstract}

There is a consensus that visual perception is characterized by two functionally independent and sequential stages. At the first stage, processing is preattentive: independent of resource allocation, all separable features are coded independently and spatially in parallel. The second stage of attentive (focal) processing requires allocation of attentional resources, which consequently cannot pass more than a limited amount of information without loss of performance (e.g., Broadbent, 1958, 1982; Neisser, 1967; Treisman \& Gelade, 1980).

Preattentive processing is commonly characterized by three basic properties: (1) it is unlimited in capacity, (2) it is spatially parallel, operating simultaneously at various locations across the visual field, and (3) it is independent of top-down control (Folk \& Egeth, 1989; Posner \& Snyder, 1975).

Evidence for the first two properties comes from visual search tasks in which a target is detected equally fast, irrespective of the number of elements in the display. Although there exists considerable disagreement over the preattentive availability of semantic identity of objects (e.g., Broadbent, 1982; Kahneman \& Treisman, 1984), it is commonly assumed that a set of primitive features or properties of objects, such as color, shape, size, brightness, and orientation, are preattentively coded in parallel. Visual search for such properties is independent of display size, suggesting unlimited and spatially parallel processing (Treisman \& Gelade, 1980). Insensitivity to perceptual load also suggests that preattentive processes operate automatically since they satisfy the loadinsensitivity criterion of automaticity. This criterion states that automatic processes are not affected when concur-

\footnotetext{
This study was supported in part by the Institute for Road Safety Research, SWOV. I thank L. C. Boer, O. Neumann, J. B. J. Riemersma, and A. F. Sanders for helpful comments on an earlier draft of the article. I would also like to thank Lester Krueger and three anonymous reviewers for their excellent comments and suggestions. Correspondence concerning this article should be addressed to Jan Theeuwes, TNO Institute for Perception, P.O. Box 23, 3769 ZG Soesterberg, The Netherlands.
}

rent information load is increased (e.g., Jonides, NavehBenjamin, \& Palmer, 1985; Neumann, 1984). The third property of preattentive processing refers to the unintentionality criterion of automaticity, which states that "automatic processes are under the control of stimulation rather than under the intentions (strategies, expectancies, plans) of the person' (Neumann, 1984, p. 258).

Recent studies suggest that this is not generally the case. Subjects can ignore salient primitive features, such as color, brightness, and form, when these features are irrelevant to their task. On the other hand, subjects appear to detect these features preattentively when these features are made relevant to the search task (Jonides \& Yantis, 1988; Theeuwes, 1990). Therefore, preattentive processing may not occur unintentionally: if relevant to the task, preattentive processing occurs; if irrelevant to the task, preattentive processing does not occur and search proceeds serially.

It should be noted that in these studies the salient feature was either task-relevant or task-irrelevant in an allor-none way: for one group of subjects, the unique feature was always irrelevant, whereas, for the other group, the feature was always relevant to the task. This condition provides the opportunity to either shut down or enable preattentive parallel search.

The question remains whether, given the occurrence of parallel preattentive processing, attention can still be strategically controlled. Given the observation that search occurs in parallel, are subjects still capable of merely attending to a task-relevant feature? In terms of properties that are thought to define automatic processing, the present experiments test whether visual search can proceed in parallel (i.e., satisfy the load-insensitivity criterion) and yet, at the same time, be selective toward the task-relevant dimension (i.e., not satisfy the unintentionality criterion of automaticity).

The present three experiments investigated selectivity between different stimulus dimensions (i.e., intensity, color, and form). Subjects viewed multielement displays $(5,7$, or 9$)$, in which two salient items, each unique in a different dimension, were simultaneously present. For 
different groups of subjects, one of the dimensions was task-relevant and the other dimension served as a distractor. The task was designed such that subjects needed to attend to the location that was cued by the task-relevant dimension. Performance when searching for a target item unique on a task-relevant dimension in the presence of an item unique on the irrelevant dimension was compared with search performance obtained in conditions in which only a single task-relevant item was present. The latter conditions are assumed to provide search functions that reflect parallel search defined as the absence of an effect of display size.

The issue investigated is whether search can be in parallel and yet be selective with respect to dimensions like color, intensity, and form. Such selectivity would provide evidence for theories, assuming that any stimulus property available at the preattentive level of processing can serve as a basis for selection (e.g., Duncan, 1980, 1981). Thus, just as advance knowledge of the position of a target provides a cue for attentional selection, advance knowledge of any other stimulus attribute (i.e., intensity, color, or form) may guide access to the limitedcapacity system. Since these theories assume that topdown control can be selectively tuned to any stimulus attribute, they predict that search for an item unique on a relevant dimension is not hindered by the presence of a feature unique on an irrelevant dimension. Given parallel search defined as the absence of an effect of display size, these theories predict the absence of an effect of a taskirrelevant distractor.

Alternatively, theories claiming that top-down selection is, first of all, based on the direction of attention to a location in visual space (e.g., Eriksen \& Yeh, 1985; Nissen, 1985; Theeuwes, 1989) would predict that selectivity within an attended area is no longer possible. These theories suggest that the extent to which interfering information can be blocked out depends on the extent to which spatial filtering is possible. Recent studies (Theeuwes, 1991; Yantis \& Jonides, 1990) suggest a role for spatial filtering, demonstrating that visual onsets and offsets cease to attract attention when subjects have the opportunity to focus their attention prior to display onset. In the present study, it is assumed that the task-relevant item can only be located by parallel processing when attention is distributed across the whole visual field. Because selectivity within spread-out beam of attention is not possible, these theories assume that a fulfillment of the load-insensitivity criterion (i.e., locating the task-relevant dimension by parallel search) also will result in fulfillment of the unintentionality criterion. In the present setup, this hypothesis predicts that any feature, relevant or irrelevant, that stands out from the background (i.e., color, intensity, or form) will attract attention and thus disrupt the processing of a task-relevant feature. Thus, according to location theories, if search is parallel, it is also unintentional. It should be noted, however, that intentional search might occur when parallel processing is shut down, resulting in serial search across the display (Theeuwes, 1990). Yet, if search is parallel, an effect of distractor type is expected, indicating that search for the task-relevant dimension is hindered by the presence of an item unique on a taskirrelevant dimension.

\section{EXPERIMENT 1}

Experiment 1 investigated selectivity between the stimulus dimensions intensity and color. The subjects viewed multielement displays in which the target line segment that determined the correct response was located in one of the display elements. For one group of subjects, the target line segment was consistently located in a display element that had an intensity that was either lower or higher than the intensity of the other elements. For the other group of subjects, the line segment was consistently located in the display element with the unique color.

\section{Method}

Subjects. Sixteen right-handed subjects, ranging in age from 18 to 24 years, participated as paid volunteers. Eight subjects were randomly assigned to the intensity condition, and $\mathbf{8}$ to the color condition. All had normal or corrected-to-normal vision and reported having no color vision defects.

Apparatus. An S-R interface with external clocks (accuracy of $1 \mathrm{msec}$ ) connected to an IBM AT-3 with video-digitizer (Matrox Inc.) controlled the timing of the events, generated video pictures, and recorded reaction times (RTs). The response panel, which was tilted $45^{\circ}$, consisted of left and right response keys $(1 \times 1 \mathrm{~cm})$, that were mounted $5.5 \mathrm{~cm}$ apart.

The stimuli appeared on a $35 \times 23 \mathrm{~cm}$ TV monitor (Conrac Model $7250 \mathrm{C} 19)$. The fixation point and target line segment were presented in white $\left(18.5 \mathrm{~cd} / \mathrm{m}^{2}\right)$ on a black background $\left(.01 \mathrm{~cd} / \mathrm{m}^{2}\right)$. The display elements consisted of outline circles that were either red or green (CIE $x y$-chromaticity coordinates of, respectively, $.616 / .357$ for red and $.282 / .561$ for green) and were matched for luminance. Their brightness was high $\left(7.5 \mathrm{~cd} / \mathrm{m}^{2}\right)$ or low $\left(1.0 \mathrm{~cd} / \mathrm{m}^{2}\right)$.

Each subject was tested in a sound-attenuated, dimly lit $2 \times 2 \times 2 \mathrm{~m}$ cubicle (Amplisilent), with his or her head resting on a chinrest adjusted to a comfortable height. The TV monitor was located at eye level, $118 \mathrm{~cm}$ from the chinrest. An intercom was used for communication with the subject.

Stimuli. The stimulus field consisted of five, seven, or nine elements, equally spaced around the fixation point on an imaginary circle whose radius was $3.4^{\circ}$. The display elements were red or green and bright or dim outline circles with a diameter of $1.4^{\circ}$, in which a $0.5^{\circ}$ line segment was placed. All display elements contained line segments that were tilted $22.5^{\circ}$ to either side of the horizontal or vertical plane. These orientations were randomly distributed in a display. In only one display element was the line segment oriented either horizontally or vertically; the orientation determined the appropriate response key (left for vertical and right for horizontal). Since the subjects responded to the horizontal or vertical orientation of a target line segment located among slightly tilted nontarget line segments, the task was assumed to require focal attention (e.g., Treisman \& Gormican, 1988), but not high spatial acuity.

Procedure. The sequence of events was as follows. Initially, a white fixation dot $\left(0.3^{\circ}\right)$ was presented at the center of the visual field. Six hundred milliseconds prior to display onset, the fixation dot increased in size to $2^{\circ}$ in order to warn the subject. The stimulus field remained present for a maximum of $4 \mathrm{sec}$ until a response was emitted. 
For different groups of subjects, the target line segment was consistently positioned either in the display element with a unique color or in the display element with a unique intensity. To prevent the development of an automatic consistently mapped (CM) detection response (see Shiffrin \& Schneider, 1977) within a block of trials, target and nontarget display elements continuously switched roles. In the intensity condition, on half of the trials, the target line was located in the sole low-intensity display element while all other elements were of a high intensity, and vice versa on the other trials. In the color condition, on half of the trials, the target line was located in the sole red display element while all other elements were green, and vice versa on the other trials. Hence, the subjects could locate the target line segment by searching for the discrepant display element within a certain dimension, rather than by searching for an item with a specific value on that dimension. In a control condition, all elements had the same intensity and the same color, and the target line segment presumably could only be located by scanning the individual elements by focal attention.

Three distractor conditions were used: (1) a neutral condition in which no distractor was present, (2) a different-dimension condition in which a distractor unique in the other dimension was present, and (3) an identical-dimension condition in which a distractor was present that was identical to the target. Figure 1 shows the various display configurations.

The intensity conditions are shown on the left side of Figure 1. Panel Al illustrates the no-distractor intensity condition in which the target line segment was always located in the display element having either a higher or lower intensity, relative to the other elements. Color was kept constant for a given trial-that is, all display elements had the same color (either red or green). Between trials the color changed randomly. Panel A2 shows the differentdimension intensity condition, which was exactly the same as the
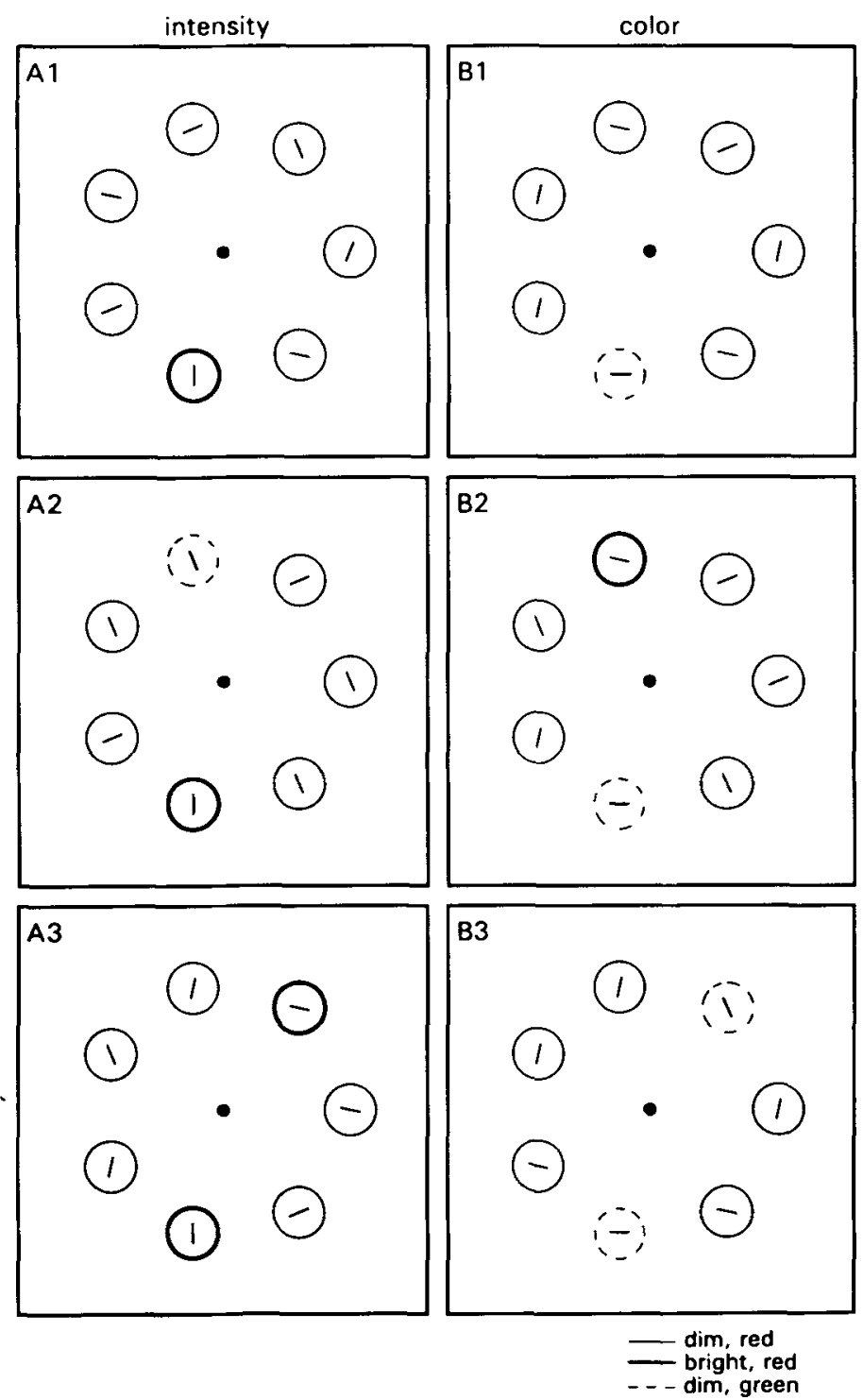

Figure 1. Examples of Display Size 7. From top to bottom each panel represents the distractor conditions no distractor, different dimension, and identical dimension, respectively. Intensity conditions are shown on the left; color conditions are shown on the right. 
condition presented in Panel A1, except that the color of one of the nontarget display elements was different from the color of the other elements. Panel A3 gives the identical-intensity condition, which was exactly the same as the condition presented in Panel Al, except that one of the nontarget display elements had the same intensity as the display element containing the target line segment The right side of Figure I presents the color condition. Panel BI gives the no-distractor color condition in which the target line segment was always located in the circle with a unique color. On each trial, all display elements had either a high or a low intensity. Between trials, the intensity changed randomly. Panel B2 gives the different-dimension color condition, which was the same as the condition presented in Panel BI, except that the intensity of one of the nontarget display elements was higher or lower than the intensity of the other elements. Panel B3 gives the identical-dimension color condition, which was the same as the condition presented in Panel B1, except that one of the nontarget display elements had the same color as the display element containing the target line segment. The target line segment appeared equally often at each of the possible locations. The position of the unique display element containing the target line segment in relation to the position of the distracting display element was randomly determined.

In the control condition, there was no unique element. The target line segment was located in one of the five, seven, or nine display elements, all of which had the same color (either red or green. randomly varied between trials) and the same intensity (either bright or dim, randomly varied between trials). The three experimental distractor conditions (no, different, and identical distractor) and the control condition were presented in separate blocks, according to a digram-balanced $4 \times 4$ Latin square. Display size was randomized within blocks. The practice session consisted of 72 trials of the nodistractor condition and 72 trials of the control condition. Each subject received each of the three distractor conditions and each con- trol condition twice in separate blocks of 144 trials. Each subject performed a total of 1,152 trials - that is, a total of 96 trials in each display-size distractor condition.

A session consisting of 144 trials lasted approximately $10 \mathrm{~min}$, with a 1-min break after 72 trials. Each block started with three dummy trials. Two subjects were run in alternating sessions. Prior to the start of the experiment, the subjects were instructed to search for the horizontal or vertical target line segment and to press the appropriate response key with one of their thumbs, which were resting on the response keys. The subjects were informed about the relation between the location of the target line segment and the unique element. It was emphasized that the subjects should fixate the central dot and not move their eyes during the course of any trial. To ensure that the subjects followed the instructions, during the practice session, eye movements were monitored on-line by means of an infrared camera. It was stressed that a steady fixation would reduce RT and make the task easier. Both speed and accuracy were emphasized. A warning beep informed the subject that an error had been committed. If no response was made after $4 \mathrm{sec}$, the trial was counted as an error. Before each session, the subjects were informed about the upcoming condition.

\section{Results}

Mean RTs and error percentages are shown in Figure 2. For each of these measures, the intensity and color conditions were subjected to separate ANOVAs, with display size and distractor condition as main factors.

In the intensity condition (Figure $2 \mathrm{~A}$ ), there was a main effect on RT for distractor type $[F(2,14)=41.2$, $p<.001]$ and an interaction between display size and distractor type $[F(4,28)=2.9, p<.05]$. In the color
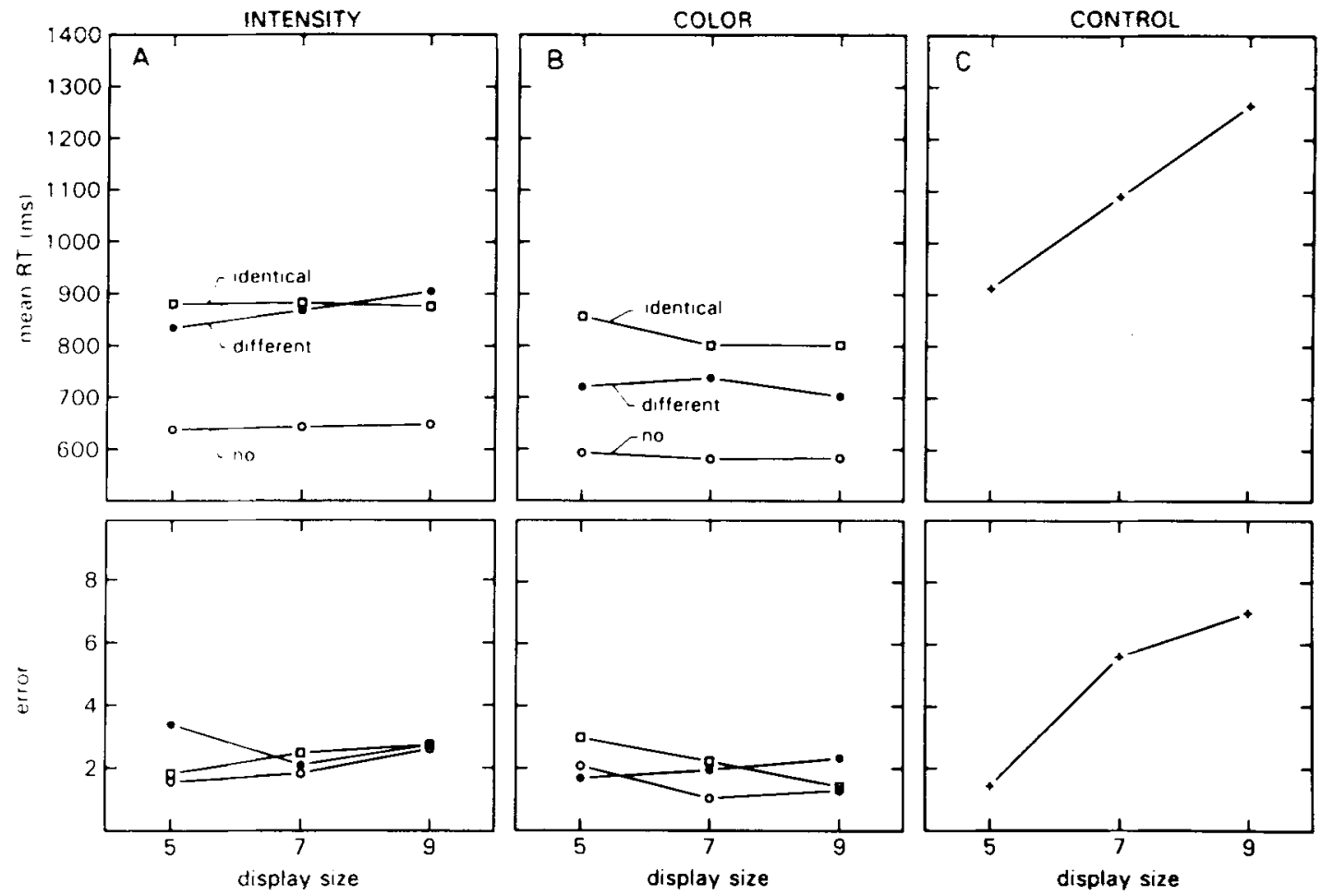

Figure 2. Experiment 1: Mean reaction times and error percentages for different distractor conditions for the intensity (Panel A), color (Panel B), and control (Panel C) conditions. 
condition (Figure 2B), there were main effects on RT for distractor type $[F(2,14)=55.0, p<.001]$ and display size $[F(2,14)=4.2, p<.05]$. In addition, the interaction between these variables was significant $[F(4,28)=$ $3.9, p<.05]$. To determine the slopes of the RT functions, the individual mean RTs were submitted to a linear regression analysis. The mean slopes for the intensity conditions were $2.7,17.7$, and $-1.1 \mathrm{msec} / \mathrm{item}$ for the no distractor, distractor in different dimension, and distractor in identical dimension, respectively. For the color condition, these figures were, respectively, $-2.5,-4.6$, and $-14.0 \mathrm{msec} / \mathrm{item}$. None of these slopes were significantly different from zero [all $t s(7)<1.04$ ], indicating parallel search across all items in the display. Inspection of the RT data reveals that search for an item with a unique intensity or unique color is distracted when an item unique on an irrelevant dimension is present. For the intensity condition, this distraction is similar to the distraction caused by the relevant dimension. For the color condition, the distraction caused by an item unique in a different dimension is somewhat less than the distraction caused by an item identical to the target.

To achieve homogeneity of the error rate variance, the mean error rates per cell were transformed by means of an arcsine transformation. For both the intensity condition and the color condition, none of the error effects were significant, which suggests that the difference in RT is not due to trading speed for accuracy.

Figure $2 \mathrm{C}$ shows the control condition collapsed over the intensity and color conditions. As is evident from the figure, when there is no unique item present in the display, both RT and errors show a clear display-size effect. The slope on RT for the control condition was $88.2 \mathrm{msec} / \mathrm{item}$. These results confirm Treisman and Gormican's (1988) claim that horizontal and vertical lines are coded as the absence of a critical feature. Since horizontal and vertical lines do not have a unique feature to distinguish them from the tilted distractors, the target line can only be detected when attention is directed serially to each item in the display.

\section{Discussion}

The finding that the search functions do not have a positive slope suggests that speeded search for the unique item is performed in parallel across all items in the display. Even in the presence of distractors, search remains essentially parallel. Therefore it can be argued that this type of search is automatic on the basis of the load-insensitivity criterion, which states that automatic processes are not hindered when concurrent information load is increased. Furthermore, the data suggest that the subjects were not capable of attending selectively to the task-relevant dimension, indicating that intentional selectivity during preattentive search is not possible.

The results favor theories stressing the role of spatial filtering. When processing preattentively, attention is divided over the whole display, which implies that intentional selectivity within this area is lost. The results pose difficulties for theories that claim that any physical stimulus attribute may be used as the basis for selection. These theories assume that selection can be based on any information that provides an increase in general knowledge about separation of signal and noise, so that there is no reason why an item unique at an irrelevant dimension would interfere with search for the relevant dimension.

Yet, the latter theories can be saved if it is assumed that, at a preattentive stage, color and intensity are not coded independently as separable features. Experiments on texture segregation suggest such conjecture. Callaghan (1984) showed that irrelevant variation of color interfered with texture segregation based on brightness and that irrelevant variation of brightness interfered with segregation based on color. Experiments on attention employing speeded classification tasks provide additional evidence: Garner and Felfoldy (1970) called color and brightness integral dimensions because these dimensions could not be processed separately.

Also, within Treisman's feature-integration theory, intensity has never been considered a separable dimension. Yet, the present data indicate that a unique intensity "pops out" from its background (i.e., target detection RT is independent of display size), suggesting that intensity is available preattentively. If the occurrence of a pop-out can be used as a diagnostic for the existence of early separable features (e.g., Treisman \& Gormican, 1988), it can be argued that intensity might be a separable feature. In this respect, it should be noted that a target with a unique color, as in Treisman's experiments, is probably different in both color and intensity, because normally a different color also has a different intensity. Yet, the observation that a unique color and a unique intensity interfere does suggest that color and intensity are not available independently at a preattentive stage.

Given these findings, the observed interferences between color and brightness might not have been entirely unexpected. Therefore, it seems important to replicate Experiment 1 using dimensions that are generally considered as separate and are assumed to be coded independently. Visual search experiments have suggested that orientation (i.e., form) and color can be distinguished at an early perceptual level, and it has been claimed that these features are registered independently by specialized modules (Treisman \& Gelade, 1980). Converging evidence comes from speeded classification tasks showing independence between form and color dimensions (Gottwald \& Garner, 1972) and from texture segregation experiments (Callaghan, Lasaga, \& Garner, 1986) showing that orientation and color are separable, rather than integral, dimensions.

\section{EXPERIMENT 2}

Experiment 2 investigated whether search can be parallel and yet be selective toward either color or form. The task was the same as in Experiment 1, except that Experiment 2 used a display in which an item had either a unique color (green or red) or a unique form (circle or 
square). The distractor conditions were comparable to those used in Experiment 1.

\section{Method}

Subjects. Sixteen new subjects, ranging in age from 17 to 25 years, participated in Experiment 2. Eight subjects were randomly assigned to the form condition, and 8 to the color condition.

Apparatus. The apparatus was identical to that used in Experiment 1 . The display elements were either red or green (same CIE $x y$-chromaticity as in Experiment 1) and had a luminance of $7.0 \mathrm{~cd} / \mathrm{m}^{2}$

Procedure. The task was similar to that in Experiment 1, except that for different groups of subjects, the target line segment was consistently positioned in a display element with a unique color or in a display element with a unique form. The display elements consisted of red or green circles $\left(1.4^{\circ}\right.$ in diameter) or squares $\left(1.4^{\circ}\right.$ on a side). The subjects were instructed to consistently search for the display element with the unique form or the unique color. Design and procedure were identical to those of Experiment 1.

\section{Results}

Mean RTs and error percentages are shown in Figure 3.

In the form condition (Figure $3 \mathrm{~A}$ ), there was only a main effect on RT for distractor type $[F(2,14)=34.8$, $p<.001]$. In the color condition (Figure 3B), there were main effects on RT for distractor type $[F(2,14)=78.3$, $p<.001]$ and display size $[F(2,14)=14.1, p<.001]$. Also, the interaction between these variables was significant $[F(4,28)=4.5, p<.01]$. The mean slopes for the form conditions were $1.2,6.0$, and $11.1 \mathrm{msec} / \mathrm{item}$ for the no distractor, distractor in different dimension, and distractor in identical dimension, respectively. For the color condition, these figures were $-2.6,-1.1$, and $-16.2 \mathrm{msec} / \mathrm{item}$, respectively. Again, none of the slopes differed from zero [all $t s(7)<1.02$ ], suggesting parallel search across all items. Planned comparisons showed that, in the color condition, the differences in response latencies between the no-distractor condition and a distractor in a different dimension was not significant $[F(1,7)=1.75$, $p=.23$ ], suggesting that search for a unique color is not hindered by a display element with a unique form. Alternatively, the same comparison for the form condition revealed a significant effect $[F(1,7)=26.6, p<.01]$, suggesting that search for a unique form is slowed down by a display element with a unique color.

The arcsine transformed error data showed that, in the color condition, none of the effects were significant, whereas, in the form condition, there was a main effect of distractor type $[F(2,14)=5.6, p<.05]$. Since this analysis indicates that error differences are nonsignificant or tend to mimic RT differences, differences in response latencies cannot be attributed to a speed-accuracy tradeoff.

Figure $3 \mathrm{C}$ shows the control condition. The slope of the control condition was $85.9 \mathrm{msec} / \mathrm{item}$, which is similar to the one found in Experiment 1.

\section{Discussion}

Again, for all search functions, RT did not increase with display size, indicating that search for a unique color or form was performed in parallel. In contrast to the results
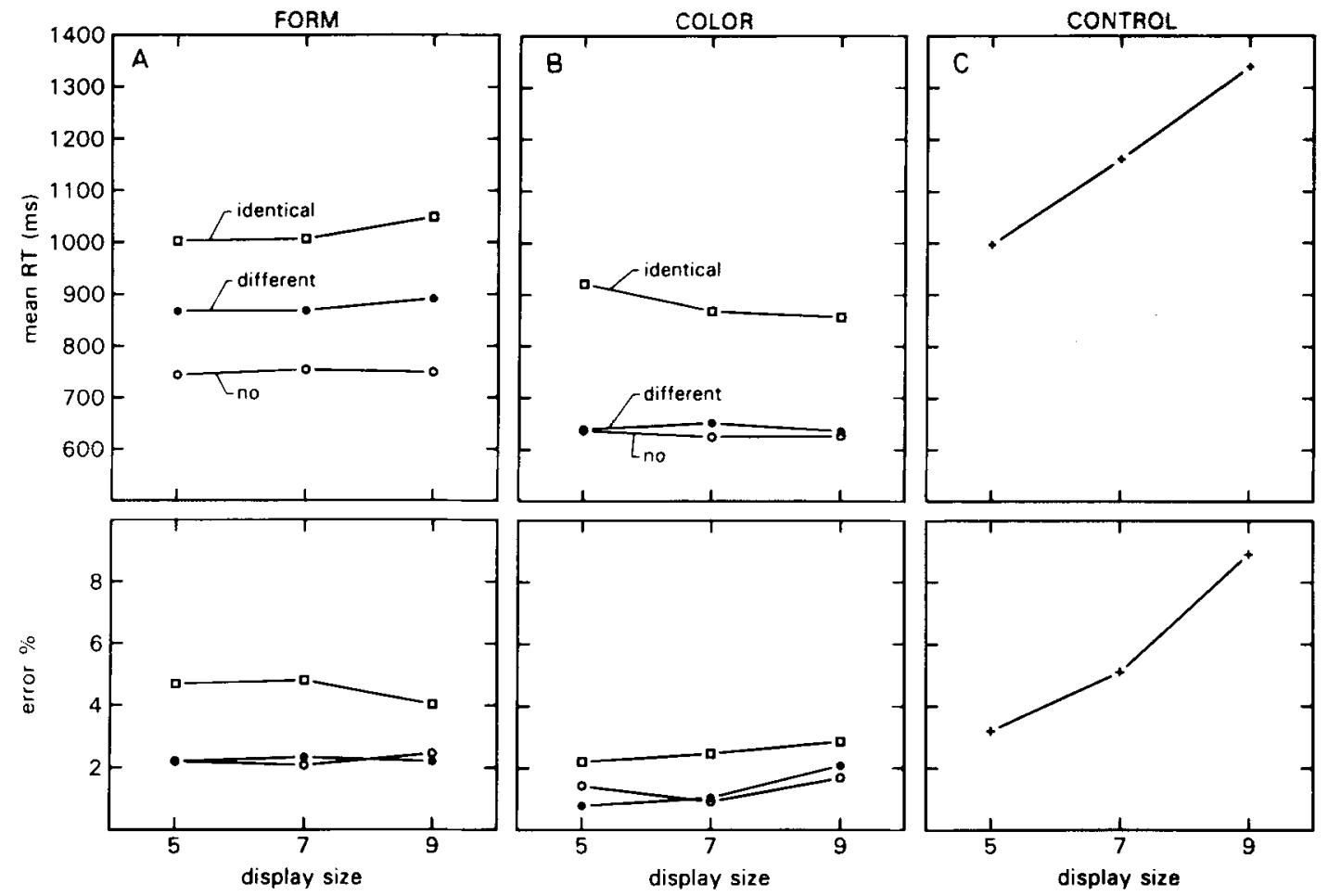

Figure 3. Experiment 2: Mean reaction times and error percentages for different distractor conditions for the form (Panel A), color (Panel B), and control (Panel C) conditions. 
of Experiment 1, perfect selective search was possible for color, since the presence of an irrelevant form had essentially no effect on speeded search for an item with a unique color. Yet, the opposite did not hold: searching for a unique form was slowed down by the presence of a unique color.

Even though there is general agreement on the separability of color and shape, the present data show that separation as such does not lead to perfect selective search: a uniquely colored item does interfere with search for a target defined by form. This is at odds with Treisman's (1988) claim that features that are analyzed by separate modules will not show any cross talk. Yet, the task in the present study differs from the usual Treisman type of "target present-absent" search tasks, in which subjects can respond on the basis of detection of activity in a particular feature map, thereby bypassing the focal attentional process (Treisman \& Gelade, 1980). In the present experiments, however, focal attention was always needed because it was not the unique feature that determined the response but the orientation of the line segment appearing in the display element defined by the unique feature. The control conditions showed that acquiring this information requires focal attention.

The hypothetical frameworks, as outlined in the introduction, did not predict the observed asymmetric selectivity between form and color. Yet, recent studies concerning texture segregation also report asymmetric interference. Pashler (1988) had subjects search for a single target that was unique in a given dimension (e.g., form or color). His Experiment 4 showed some interference when subjects searched for a target that differed in form (either $\mathrm{O}$ or $/$ ) from background elements whose color varied irrelevantly (green vs. red). Search was not affected when subjects searched for a color difference among distractors that varied in form (his Experiment 3). Since the asymmetric interference was rather small and did not occur in all experiments, Pashler (1988) hardly discusses the implications of this asymmetry. However, texture segregation experiments of Callaghan (1989) confirm the asymmetry between form and color. When boundary judgments were based on form differences, segmentation was significantly impaired by color variation. However, color segmentations were not affected by form variation.

For the present study, one might consider (admittedly post hoc) a model of performance that can account for the asymmetry. Assume that attention is divided over the whole display and that the preattentive process computes in parallel and independently only differences in features according to a simple horse race. Subsequently, the focal attention needed for identification is directed serially in the order of the availability of the difference signals at each location in the display. The difference signal represents an activation at a particular location, which depends on the difference within a particular dimension (e.g., color or form) between the display element at that location and each of the other display elements. The differ- ence signal is similar to the bottom-up activation in the parallel stage of Cave and Wolfe's (1990) guided search model. Earlier studies (Quinlan \& Humphreys, 1987) have shown that color information becomes available earlier than does shape information, a finding which is in line with the present data. In the no-distractor conditions, the mean RT averaged over display size for the form and color conditions were 750 and 630 msec, respectively, suggesting that in these conditions the difference in color is available about $120 \mathrm{msec}$ earlier than is the difference in form. When one assumes that attention is captured by the first available feature, the unique color gets focal attention first and, consequently, performance is not affected by the presence of a unique form. Alternatively, when searching for a unique form, attention is unintentionally captured by the earlier available difference in color, resulting in an increase in RT, which is independent of display size. Crucial for the present model is that attention is unintentionally switched based on the order of the availability of the local difference in features, along with the conjecture that features that are relevant to the task do not receive a higher priority. Indeed, the model assumes that after the completion of the first parallel stage in which separable features are analyzed independently, no intentional selectivity is possible at the second stage of focal attention.

The finding that a distractor that is identical to the target causes more interference than does a differentdimension distractor is in agreement with the model outlined above. Preattentively, features of different dimensions can be coded independently, suggesting at least interference-free processing at the parallel stage. Yet, as Treisman (1982) pointed out, even when a feature is locally unique and surrounded by contrasting items, a preattentive pop-out is precluded when the same feature is also present elsewhere in the display. Thus, the identical distractor causes not only interference at the second stage of focal attention but also at the first preattentive stage.

The results of Callaghan (1989) on texture segregation, however, do not support the horse-race model above. Even though Callaghan admits that there is no apparent mechanism that can explain the asymmetry, her data show that even when form differences are easier to discriminate than color differences (mean RT of $571 \mathrm{msec}$ for "form" control condition vs. mean RT of $592 \mathrm{msec}$ for "hue hard" control condition), the asymmetry in interference remains.

\section{EXPERIMENT 3}

Experiment 3 was designed to test the horse-race model outlined above. To alter the availability in time of the local differences in color and form features, color discrimination was made harder and form discrimination was made easier. If attention is switched on the basis of the order of the availability of the local feature, it is expected that the asymmetry also will switch, so that form, and not color differences, produce interference. Yet, if the 
unique color continues to interfere even when it is harder to discriminate, the results would confirm Callaghan's (1989) findings.

\section{Method}

Subjects. Eight new subjects, ranging in age from 17 to 25 years, participated in Experiment 3.

Apparatus. The apparatus was identical to that used in Experiment 1.

Procedure. The task was similar to that in Experiment 2. In comparison to Experiment 2, the differences in color were less salient (CIE $x y$-chromaticity coordinates of, respectively, .440/.488 for red and $.400 / .516$ for green) and were matched for luminance $\left(7.0 \mathrm{~cd} / \mathrm{m}^{2}\right)$. The form differences were increased by changing the square into a diamond form (width $1.2^{\circ}$, height $2^{\circ}$ ), making the diamond easier to tell apart from the circles.

In comparison to the previous experiments, color and form conditions were varied within subjects to allow more sensitive comparisons between conditions. Since the identical-dimension distractor condition is not relevant for testing the hypotheses discussed above, this condition was omitted. For form and color conditions, there were two distractor conditions (no distractor and different dimension), which were presented according to a digram Latin square. Each subject received these four conditions in separate sessions.

A session consisted of 72 practice trials, followed by four blocks of 72 experimental trials. Before each session, the subjects were informed about the relationship between the location of the target line segment and the unique display element; they were instructed to use this information. Between blocks of trials there was a 1-min break. Each block started with three dummy trials. Each subject performed a total of 96 trials in each display-size distractor condition.

\section{Results}

Mean RTs and error percentages are shown in Figure 4. The individual mean RTs were submitted to an ANOVA, with dimension (form and color), display size (five, seven, and nine), and distractor as factors. There was a main effect on RT for both display size and distractor $[F(2,14)=5.9, p<.05$, for display size, and $F(1,7)=24.0, p<.01$, for distractor]. Also, the interaction between these variables was significant $[F(2,14)$ $=4.2, p<.05$ ]. In addition, the interaction between dimension and display size was significant $[F(2,14)=7.4$, $p<.01]$. For the form condition, the mean slopes were -2.5 and $1.2 \mathrm{msec} / \mathrm{item}$ for no distractor and different dimension, respectively. For the color condition, the slopes were 9.0 and $22.8 \mathrm{msec} /$ item, respectively. None of the slopes differed significantly from zero [all $t s(7)<1.37$ ], suggesting parallel search across all items. The overall analyses suggest, as evident in Figure 4 , that the main effect of display size is primarily due to an increase in RT in the color condition. Planned comparisons showed, for the color condition, a significant difference between the mean RTs of the no-distractor condition and the distractor-in-a-different-dimension condition $[F(1,7)=$
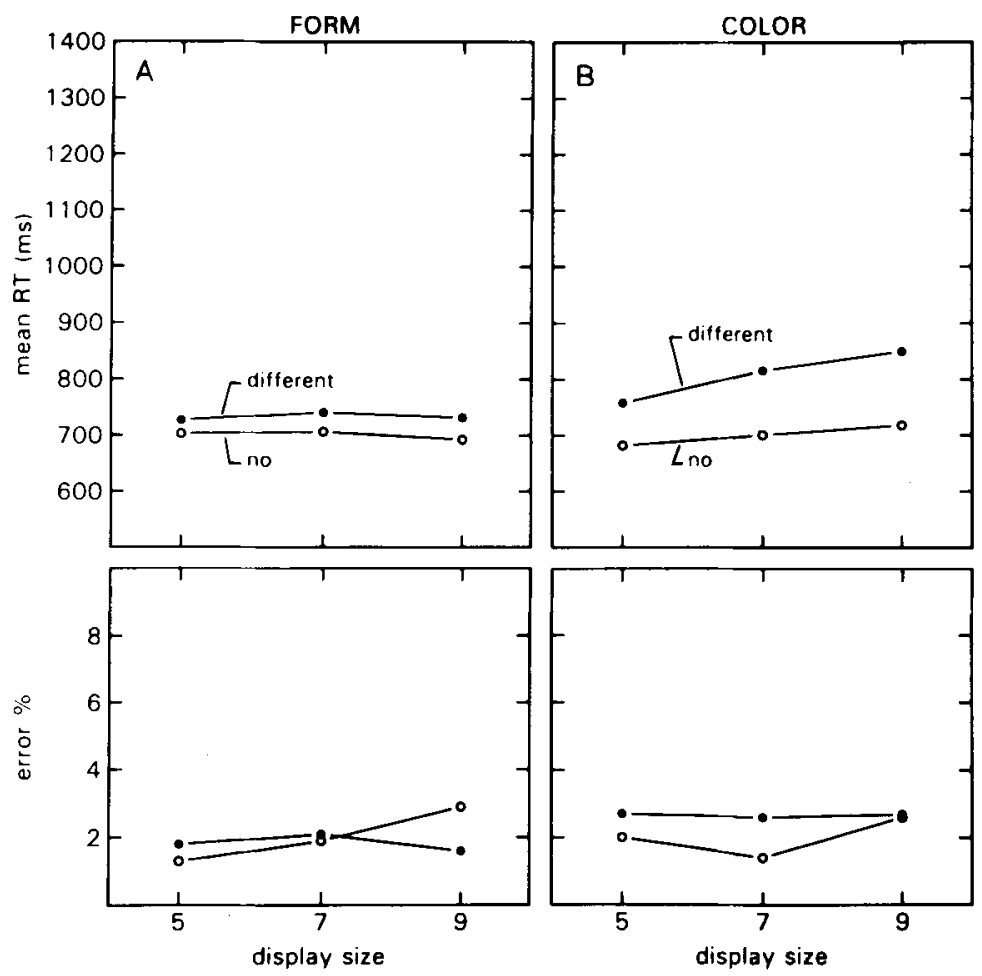

Figure 4. Experiment 3: Mean reaction times and error percentages for the different distractor conditions for the form (Panel A) and color (Panel B) conditions. 
$19.5, p<.01]$, implying that search for a unique color is slowed down by the presence of an item with a unique form. In addition, the same comparison for the form condition revealed that search for a unique form is not reliably affected by the presence of a unique color $[F(1,7)=$ $3.1, p=.12]$.

The arcsine transformed error data showed that none of the effects were significant, suggesting that differences in response latencies are not due to a speed-accuracy tradeoff.

\section{Discussion}

Again, all search functions are essentially flat, suggesting that search was performed in parallel. Albeit the color difference is relatively small, search still can be performed in parallel. Yet, a further reduction of the color differences would probably lead to serial search because, as evident in Figure 4, a display-size effect starts to emerge. Contrary to Experiment 2, the presence of a unique form had a large effect on speeded search for the unique color. On the other hand, search for a unique form was only marginally affected by the presence of a unique color.

The finding that the asymmetry between the color and form dimensions is dependent on the relative discriminability of each dimension is not in accordance with Callaghan's (1989) findings on texture segregation. In these experiments, it was shown that color continues to interfere even when color is harder to discriminate than form. Yet, the present findings are not completely in accordance with the horse-race model outlined earlier. The mean RTs for the no-distractor conditions of color and form are not different (701 msec for form and $700 \mathrm{msec}$ for color), suggesting that the difference in form is detected equally as fast as the difference in color. If attention is switched on the basis of the order of the availability of the difference signal at each location, comparable interferences for color and form would have been expected. The present data indicate that search for a unique color was indeed significantly affected by the presence of a unique form. Yet, search for a unique form was only marginally affected by the presence of the unique color.

\section{GENERAL DISCUSSION}

The present experiments were designed to explore the limits of perceptual selectivity. Given the assertion of preattentive parallel search, the present study investigated selective allocation of attention to task-relevant stimulus dimensions. Two classes of theory provided opposing predictions. First, theories claiming that attention selects objects in their locations predict that a salient object at that location-task-relevant or task-irrelevant-will attract attention. Alternatively, theories claiming that selection can be based upon any stimulus property predict that attention can simply be tuned to a particular task-relevant stimulus dimension. Although the results do not completely support either class of theory, the results favor the first hypothesis. The asymmetric selectivity between color and form is not due to the intentions of the subjects, but reflects differences in preattentive processing time of these particular features.

With respect to automaticity, the lack of a display-size effect suggests that search for a unique color, intensity, or form does satisfy the load-insensitivity criterion of automaticity, even when distractor items are present. Since the observed selectivity is not due to the intentions of the subject, it can be argued that the unintentionality criterion is also fulfilled (i.e., during preattentive processing, selection is completely under the control of stimulation).

The present data seem to contradict the conclusions of Pashler (1988) concerning texture segregation. In his experiments, subjects searched for a single target that was unique in a given dimension (e.g., form or color). For example, subjects searched for a specific form (either $\mathrm{O}$ or /) within a display of 90 elements, all of which had the same color or had different colors. The results showed minimal interference when the other dimension (in this example, color), known to be irrelevant, was randomly varied. Pashler (1988) claimed that his results argued against a single difference-detection process that operates on multidimensional levels, suggesting that subjects are capable of selectively allocating attention to task-relevant stimulus dimensions. In addition, Treisman (1988) reports similar results for visual search: heterogeneous variation across dimensions of nontargets (e.g., color and orientation) hardly has an effect on search times. For example, search time for a horizontal line segment among vertical line segments of mixed colors (red, green, white, and blue) is similar to the search time for a horizontal line segment among vertical lines of a single color (green). Treisman claims that subjects simply can check for the activity signaling a contrasting item in the relevant targetdefining module and ignore the others.

The present findings do not support such selectivity: subjects lack the ability to simply ignore the irrelevant dimension. Yet, studies cited above and the present study used different manipulations of the irrelevant dimension. In the present study, one item was unique within the irrelevant dimension (e.g., one green among all red items), whereas, in the above-cited studies, there was never a single unique item. Within a display, items had a particular value within the irrelevant dimension (e.g., some items were red, green, purple, blue, etc.). If one adheres to the model outlined earlier, which assumes that the preattentive process calculates for each dimension and each location the difference between an element at that particular location and each of the other elements, random variation within a dimension will not give particularly high local activations. Because none of the elements within the irrelevant dimension has a high activation, top-down selectivity for the relevant dimension is hardly affected. Yet, a single item unique within the irrelevant dimension will cause a relatively high activation and is expected to affect performance, a finding which is supported by Pashler's (1988) Experiments 6 and 7. These experiments 
showed large interference effects (both in percentage correct and in response latencies) for the detection of a target defined by form when a single irrelevant item with a unique color was present, a result which is in accordance with the present findings.

In short, the results suggest that when search is performed preattentively in parallel, top-down selectivity toward a specific stimulus dimension is not possible. If preattentive search is automatic in the sense of the loadinsensitivity criterion, it is also automatic in the sense of the unintentionality criterion. It was speculated that stimulus dimensions are processed in parallel and that attention is captured by the feature that is available first. When there is a large difference in preattentive processing time between stimulus attributes, unintentional asymmetrical selectivity appears to be obtained.

\section{REFERENCES}

Broadbent, D. E. (1958). Perception and communication. London: Pergamon

Broadbent, D. E. (1982). Task combination and the selective intake of information. Acta Psychologica, 50, 253-290.

Callaghan, T. C. (1984). Dimensional interaction of hue and brightness in preattentive field segregation. Perception \& Psychophysics, 36, 25-34.

Callaghan, T. C. (1989). Interference and dominance in texture segregation: Hue, geometric form, and line orientation. Perception \& Psychophysics, 46, 25-34.

Callaghan, T. C., Lasaga, M. I., a Garner, W. R. (1986). Visual texture segregation based on orientation and hue. Perception \& Psychophysics, 39, 32-38.

CAVE, K. R., Wolfe, J. M. (1990). Modeling the role of parallel processing in visual search. Cognitive Psychology, 22, 225-271.

DUNCAN, J. (1980). The locus of interference in the perception of simultaneous stimuli. Psychological Review, 87, 272-300.

Duncan, J. (1981). Directing attention in the visual field. Perception \& Psychophysics, 30, 90-93.

ERIXSEN, C. W., YEH, Y. Y. (1985). Allocation of attention in the visual field. Journal of Experimental Psychology: Human Perception \& Performance, 11, 583-597.

Folk, C. L., EgETh, H. (1989). Does the identification of simple features require serial processing? Journal of Experimental Psychology: Human Perception \& Performance, 15, 97-110.

GARNER, W. T., Felfoldy, G. L. (1970). Integrality of stimulus dimensions in various types of information processing. Cognitive Psychology, 1, 225-241

GoTTWALD, R. L., \& GARNER, W. R. (1972). Effects of focusing strategy on speeded classification with grouping, filtering, and condensation tasks. Perception \& Psychophysics, 11, 179-182.
Jonides, J., Naveh-Benjamin, M., \& Palmer, J. (1985). Assessing automaticity. Acta Psychologica, 60, 157-171.

JoNidES, J., \& YANTIS, S. (1988). Uniqueness of abrupt visual onset in capturing attention. Perception \& Psychophysics, 43, 346-354.

Kahneman, D. \& Treisman, A. (1984). Changing views of attention and automaticity. In R. Parasuraman \& D. R. Davies (Eds.), Varieties of attention (pp. 29-61). New York: Academic Press.

Neisser, U. (1967). Cognitive Psychology. New York: AppletonCentury-Crofts.

Neumann, O. (1984). Automatic Processing: A review of recent findings and a plea for an old theory. In W. Prinz \& A. F. Sanders (Eds.), Cognition and motor processes (pp. 255-290). Berlin: Springer-Verlag

Nissen, M. J. (1985). Accessing features and objects: Is location special? In M. I. Posner \& O. S. M. Marin (Eds.), Attention and performance $X I$ (pp. 205-219). Hillsdale, NJ: Erlbaum.

PASHler, H. (1988). Cross-dimensional interaction and texture segregation. Perception \& Psychophysics, 43, 307-318.

POSNER, M. I., \& SNYDER, C. R. R. (1975). Attention and cognitive controls. In R. L. Solso (Ed.), Information processing and cognition: The Loyola symposium (pp. 55-85). Hillsdale, NJ: Eribaum.

Quinlan, P. T., \&umphreys, G. W. (1987). Visual search for targets defined by combinations of color, shape, and size: An examination of the task constraints on feature and conjunction searches. Perception \& Psychophysics, 41, 455-472.

Shiffrin, R. M., SCHNEIDER, W. (1977). Controlled and automatic human information processing. II: Perceptual learning, automatic attending and a general theory. Psychological Review, 84, 127-190.

TheEUWES, J. (1989). Effects of location and form cuing on the allocation of attention in the visual field. Acta Psychologica, 72, 177-192.

Theeuwes, J. (1990). Perceptual selectivity is task dependent: Evidence from selective search. Acta Psychologica, 74, 81-99.

THEEUWES, J. (1991). Exogenous and endogenous control of attention: The effect of visual onsets and offsets. Perception \& Psychophysics, 49, 83-90.

Treisman, A. M. (1982). Perceptual grouping and attention in visual search for features and for objects. Journal of Experimental Psychology: Human Perception \& Performance, 8, 194-214.

Treisman, A. M. (1988). Feature and objects: The fourteenth Bartlett memorial lecture. Quarterly Journal of Experimental Psychology, 40, 201-237.

Treisman, A. M., Gelade, G. (1980). A feature integration theory of attention. Cognitive Psychology, 12, 97-136.

Treisman, A. M., Gormican, S. (1988). Feature search in early vision: Evidence from search asymmetries. Psychological Review, 95 , $15-48$.

YANTIS, S., \& JonIDES, J. (1990). Abrupt visual onsets and selective attention: Voluntary versus automatic allocation. Joumal of Experimental Psychology: Human Perception \& Performance, 16, 121-134.

(Manuscript received October 12, 1990; revision accepted for publication March 11, 1991.) 